\title{
Preface
}

\section{Special Issue on M\&M2008}

Annual conference of the Materials \& Mechanics Division in the Japan Society of Mechanical Engineers[JSME] "M\&M2008" was held on September 16-18, 2008, at Ritsumeikan University, Kusatsu. A number of presentations in a wide range of this division had been made in 15 organized sessions and 10 general sessions. Several joint programs such as panel discussion, special forums and tutorial for young researchers were also organized together with poster sessions and exhibition of mechanical products.

With respect to the above meeting of M\&M2008, publication of a special issue in the Journal of Solid Mechanics and Materials Engineering was approved by the headquarters of the JSME. Thus, editorial committee was organized by the following members; T. Fukuoka(Kobe University), M. Hojo (Kyoto University), S. Imatani(Kyoto University), H. Inoue(Tokyo Institute of Technology), M. Kawai(University of Tsukuba), H. Kobayashi(Osaka University), Y. Nakai(Kobe University), T. Nakamura(Hokkaido University), F. Narita(Tohoku University), S. Ogata(Osaka University), T. Sakai(Ritsumeikan University), T. Sawa(Hiroshima University), N. Takano(Keio University), K. Tanaka(Doshisha University) and S. Ueda(Osaka Institute of Technology). "Call for Papers" was indicated in the URL of the JSME and it was sent to every speaker in the present conference of M\&M2008.

Extended papers were submitted to the special issue for the benefit of the wider international community in the area of the Materials and Mechanics. Through the strict reviewing, 18 papers including one review paper were finally accepted in this publication. On behalf of the editorial committee, we would like to express our sincere thanks to the authors and a number of reviewers as well as editorial committee members for their kind contributions to publish this meaningful journal.

\section{Guest Co-Editors}

Tatsuo SAKAI, Professor

College of Science and Engineering

Ritsumeikan University

1-1-1 Nojihigashi, Kusatsu, Shiga, 525-8577 JAPAN

Naoki TAKANO, Professor

Faculty of Science and Technology

Keio University

3-14-1 Hiyoshi, Kohoku-ku, Yokohama, Kanagawa, 223-8522 JAPAN 